REVISTA INTERNACIONAL DE CIENCIAS DEL DEPORTE International Journal of Sport Science

Rev. int. cienc. deporte

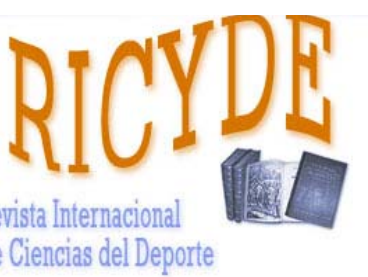

International Journal of Sport Science VOLUMEN IX - AÑO IX

Páginas:23-37 ISSN:1885-3137

No 31 - Enero - 2013

\title{
Las características laborales del profesorado de las actividades físico-deportivas extraescolares en función de la edad y el sexo, dentro de la Comunidad de Madrid \\ The employment characteristics of teachers of extracurricular sport activities according to their age and gender in the Community of Madrid
}

\author{
Jana María Gallardo-Pérez \\ Universidad Politécnica de Madrid \\ María Espada-Mateos \\ Universidad Pontificia de Comillas \\ José Antonio Santacruz-Lozano \\ Ángel Clemente-Remón \\ Universidad Alcalá
}

\begin{abstract}
Resumen
En relación a la población de las personas que desarrollan la función de docencia de las actividades físico-deportivas extraescolares en la Comunidad de Madrid, los objetivos del presente estudio pretenden describir y analizar la relación entre los tipos de contratos, los ingresos y las variables sociodemográficas. Esta investigación sigue una metodología cuantitativa, de corte transversal y descriptiva en una muestra de 350 personas. El muestro ha sido aleatorio por conglomerados aplicándose una entrevista estandarizada por medio de cuestionario. Los resultados del estudio muestran que existe relación significativa entre el tipo de contrato y la edad $[\times 2(6)=17.462 ; p=.008 ; \Phi=.616]$, la experiencia profesional y el sexo $[x 2(36)=79.047 ; p=.000 ; \Phi=.475]$, los ingresos con el tipo de centro educativo donde se realiza la actividad $[x 2(14)=185.922 ; p=.000 ; \Phi=.745]$ y el sexo $[x 2(7)=15.943 ; p=.026$; $\Phi=.218]$. Las conclusión final del estudio indica que la situación profesional de las personas que trabajan en este ámbito es bastante inestable y precaria.
\end{abstract}

Palabras clave: actividad física extraescolar; contratos; salario; sexo; edad.

\section{Abstract}

In regard to the group of people carrying out the function of teaching extracurricular sport activities in the Community of Madrid, the aims of this study are to describe and analyse the relationship among contract, salary and demographic variables. The present study used a quantitative method. A cross-sectional study was performed by means of face-to-face interviews to a random sample of 350 teachers that completed a brief questionnaire. The results show a significant relationship between type contract and age $[\times 2(6)=17.462 ; p=.008 ; \Phi=.616]$, professional experience and gender $[\times 2(36)=79.047 ;$ $\mathrm{p}=.000 ; \Phi=.475]$, salary with type of school $[\mathrm{x} 2(14)=185.922 ; \mathrm{p}=.000 ; \Phi=.745]$ and gender $[\mathrm{x} 2$ $(7)=15.943 ; p=.026 ; \Phi=.218]$. The general conclusión suggests that the professional situation of the people who work in this area is quite unstable and precarious.

Key words: extracurricular sport activities; contract; salary; gender; age.

Correspondencia/correspondence: Jana María Gallardo-Pérez

Facultad de Ciencias de la Actividad Física y del Depore-IEF. Universidad Politécnica de Madrid Calle Martín Fierro No 7, Ciudad Universitario, 28040, Madrid. España

E-mail: janam.gallardo@upm.es

Recibido el 22 de febrero de 2012; Aceptado el 27 de septiembre de 2012 
Gallardo-Pérez, J.M.; Espada-Mateos, M.; Santacruz-Lozano, J.A.; Clemente-Remón, A. (2013). Las características laborales del profesorado de las actividades físico-deportivas extraescolares en función de la edad y el sexo, dentro de la Comunidad de Madrid. RICYDE. Rev. int. cienc. deporte. 30(9), 23-37. http://dx.doi.org/10.5232/ricyde2013.03102

\section{Introducción}

$\mathrm{L}$ a situación socioeconómica en España ha cambiado mucho en los últimos 50 años, así, se puede observar que el deporte, entendido como competición, espectáculo, alto rendimiento, etc., y la actividad física, dentro de la cual se engloba, salud, ocio y recreación, y en ambos casos se incluyen la docencia y el entrenamiento, hacen ver que existen varios campos profesionales de actuación dentro de este ámbito (Carratalá, Mayorga, Mestre, Montesinos y Rubio, 2011).

La diversificación dentro del mundo deportivo a nivel del mercado laboral marca los nuevos conceptos de actividad física y deporte. Apareciendo nuevos campos profesionales, como por ejemplo la gestión, la recreación, la salud, el mundo del espectáculo y la alta competición (Martínez, 1992; Camerino y col., 1995, citados en Sánchez y Rebollo, 2000). Para poder mejorar la calidad del mercado laboral deportivo, hay que conocer los factores de elección del campo profesional y los perfiles profesionales de las personas que trabajan en el mismo. De esta manera los factores de elección del campo profesional son: el vocacional, que supone dar una respuesta a diferentes situaciones motivacionales propias; el factor de estabilidad laboral, que condiciona principalmente la elección del campo profesional posterior y por último, el factor retribución económica, ya que de éste se desglosa la oportunidad de tener unos ingresos más altos. De esta forma los cuatro ámbitos de actuación dentro de la actividad física y el deporte serían los siguientes, según Sánchez y Rebollo (2000): Enseñanza- Investigación, Gestión-Recreación, Entrenamiento deportivo y Actividad física y salud.

Si centramos esta investigación en el campo profesional del entrenamiento deportivo, donde estarían contempladas las actividades físico-deportivas extraescolares, podemos explicar el perfil profesional del entrenador, basándonos en las características expuestas por Carratalá (2011), y Delgado-Noguera (2000), tras realizar una exhaustiva revisión bibliográfica:

- Actuar con integridad.

- Crear un clima positivo.

- Apoyar a los participantes dentro y fuera del equipo.

- Tomar decisiones y establecer normas.

- Dirigir las sesiones de entrenamiento.

- Adquirir la formación necesaria y actualizar sus conocimientos.

- Fomentar la deportividad.

- Recordar la finalidad de diversión del deporte.

- Saber dirigir y hacer las críticas hacia sus alumnos.

- Recordar que existen otros intereses y compromisos a parte del deporte.

A su vez, debido a que ofrecen una gran influencia y repercusión en sus alumnos, deben tener objetivos específicos en su formación, como son, según Carratalá (2011):

- Gran conocimiento del deporte que imparten.

- Actuación a través de criterios pedagógicos.

- Imparcialidad en las acciones de sus alumnos.

- Conocer las características físicas, psicológicas y sociológicas de sus alumnos.

- Utilización y buen uso de las técnicas de motivación y comunicación.

- Planificación y programación de la clase.

- Evaluación y control de la clase. 
Gallardo-Pérez, J.M.; Espada-Mateos, M.; Santacruz-Lozano, J.A.; Clemente-Remón, A. (2013). Las características laborales del profesorado de las actividades físico-deportivas extraescolares en función de la edad y el sexo, dentro de la Comunidad de Madrid. RICYDE. Rev. int. cienc. deporte. 30(9), 23-37. http://dx.doi.org/10.5232/ricyde2013.03102

A continuación, se expone la situación en la que se encuentran estos profesionales, es decir, el mercado laboral donde trabajan. De esta forma, Madella (2003), expone que para poder analizar el mercado laboral de actividad física y deporte existen diferentes metodologías, por lo que a la hora de comparar los datos obtenidos pueden surgir ciertos problemas. Además, este mismo autor manifiesta que todavía hay algunos países que no contemplan el empleo de actividad física y deporte dentro de la legalidad del mercado laboral.

A pesar de estos problemas, existen diferentes estudios como el de Camy (2006), a nivel europeo, y los de Martínez del Castillo (1991), De Diego y col., (2004), Campos (2005), Martínez (2007) y Martínez y col., (2008) a nivel nacional, que analizan diferentes aspectos del mercado laboral de actividad física y deporte. Además, existen otros estudios como el de Álamo (2002, 2004), Nuviala (2002) y González (2008a) que analizan este aspecto de forma más específica, ya que analizan el mercado laboral del deporte escolar.

Uno de los aspectos que se desprenden de estas investigaciones es que existe un alto porcentaje de personas que no tienen ningún tipo de contrato. Así, en el estudio de Martínez del Castillo (1991), las personas que trabajaban en funciones de actividad física y deporte sin contrato representaban un $16 \%$. En lo referente a las actividades físico-deportivas extraescolares, Campos (2005) obtuvo un dato muy significativo e importante, y es que más del $50 \%$ de las relaciones laborales de las personas que trabajan en actividades físicodeportivas extraescolares en centros educativos representan situaciones laborales en las que no hay ningún tipo de contratación.

Por su parte, Nuviala (2002) expone que la situación laboral de los entrenadores en el deporte escolar es precaria, debido a que la mayoría de ellos sólo perciben una gratificación económica, siendo solamente el $31 \%$ de los técnicos los que tienen un contrato laboral.

Igualmente, en relación a la temporalidad de los contratos, Campos (2005) determina que la tipología de los contratos de las personas que trabajan en la función de docencia de actividad físico-deportiva extraescolar es mayoritariamente contratación temporal, ya que más del $90 \%$ de las personas que trabajan en actividades físico-deportivas extraescolares en centros educativos tienen este tipo de contratación y, solamente menos del $10 \%$ son personas que poseen una contratación indefinida. Asimismo, González (2008a) expone que la gran mayoría de las personas que trabajan como docentes de actividades físico-deportivas extraescolares y tienen contrato, poseen contratos de carácter temporal $(83,4 \%)$, siendo sólo un $16,6 \%$ las personas que tienen un contrato de tipo indefinido.

A su vez, Fraile (1996) determina que la situación de empleo para los monitores deportivos es bastante precaria, pues la mayoría están bajo contratos temporales. Además, en el 75\% de los casos sus ingresos son inferiores a 300 euros. Al respecto, Nuviala (2002) concluye que la compensación económica por hora de trabajo es desigual, ya que puede variar entre 3 y más de 9 euros la hora.

Asimismo, el 63,2\% de las personas trabajan en otras funciones laborales, además de en la docencia en el deporte escolar, por lo que la mayoría de los entrenadores no tienen como principal actividad profesional el deporte escolar (Álamo y col., 2002). Además, el 47,4\% no recibe retribución económica mensual por realizar dicha función (Álamo, 2004).

Por otro lado, la movilidad laboral en este ámbito es alta, y así se refleja en el estudio de Campos (2005), donde se obtiene que más del 70\% de estas personas llevan desarrollando este trabajo en su entidad actual menos de 3 años, y menos del $30 \%$ de las personas 
Gallardo-Pérez, J.M.; Espada-Mateos, M.; Santacruz-Lozano, J.A.; Clemente-Remón, A. (2013). Las características laborales del profesorado de las actividades físico-deportivas extraescolares en función de la edad y el sexo, dentro de la Comunidad de Madrid. RICYDE. Rev. int. cienc. deporte. 30(9), 23-37. http://dx.doi.org/10.5232/ricyde2013.03102

consideran que este tipo de trabajo no es temporal. En este sentido, Saura (1996) determina que el $23,5 \%$ de los entrenadores llevan realizando su función como entrenador tan sólo desde hace un año o menos.

Por otro lado, Montesinos (2005) explica, que debido a la precaria situación laboral de las personas que trabajan en actividades físico-deportivas extraescolares en centros educativos, es difícil garantizar una práctica eficaz, segura, saludable y educativa para el alumnado de los propios centros educativos, cuestión que deben analizar tanto los agentes y entidades implicadas como las diferentes administraciones públicas.

Igualmente, el informe realizado por Comisiones Obreras (2000) determina que la percepción generalizada de los trabajadores es la de estar inmersos en un sector caracterizado por la economía sumergida, facilitada de nuevo por la ausencia de convenios reguladores. Las condiciones de contratación y remuneración son fijadas por los empresarios, y éstas no llegan a satisfacer las necesidades de los monitores o profesores.

Al respecto, diferentes autores como Mosquera, Rivas y Saavedra (1998), Gómez (2001), Garrigós (2002), Campos (2005) y González (2008a) coinciden en que esta situación produce una gran desprotección laboral y social del trabajador. Además, señalan que la hacienda pública deja de recibir importantes ingresos económicos, sin olvidar que dicha situación supone un menor reconocimiento de las profesiones y de los profesionales de actividad física y deporte, y evita la transformación de estos empleos ocultos en otros con mejores condiciones laborales. Según Campos (2001), todo ello tiene lugar ante la pasividad y/o complicidad de la Administración pública.

\section{Objetivos}

- Conocer y describir las características laborales del profesorado de actividad físicodeportiva extraescolar de la Comunidad de Madrid, en función de la edad y el sexo de la población objeto de estudio.

- Describir el tipo de contratación de las personas que trabajan en funciones laborales de docencia de actividad físico-deportiva extraescolar en la Comunidad de Madrid.

- Analizar la experiencia profesional de las personas que trabajan en funciones laborales de docencia de actividad físico-deportiva extraescolar dentro de las entidades empleadoras pertenecientes a la Comunidad de Madrid.

- Conocer los ingresos mensuales de las personas que trabajan en funciones laborales de docencia de actividad físico-deportiva extraescolar en la Comunidad de Madrid, en función del centro educativo y del sexo.

\section{Método}

La investigación ha seguido una metodología cuantitativa de corte descriptivo (Alvira, 2002). En el desarrollo de la metodología, los procedimientos que se han seguido son los propios de la encuesta, ya que se ha realizado la aplicación de un procedimiento estandarizado de recogida de la información mediante preguntas sobre la muestra de población estudiada (Lussier \& Kimball, 2008).

\section{Selección de la población y muestra}

La población objeto de estudio han sido las personas que desarrollan la función de docencia de las actividades físico-deportivas extraescolares en los centros educativos de Educación 
Gallardo-Pérez, J.M.; Espada-Mateos, M.; Santacruz-Lozano, J.A.; Clemente-Remón, A. (2013). Las características laborales del profesorado de las actividades físico-deportivas extraescolares en función de la edad y el sexo, dentro de la Comunidad de Madrid. RICYDE. Rev. int. cienc. deporte. 30(9), 23-37. http://dx.doi.org/10.5232/ricyde2013.03102

Secundaria de la Comunidad de Madrid.

Para establecer el universo objeto de estudio, el número de personas que desarrollan la función de docencia en las actividades físico-deportivas extraescolares en los centros educativos de Educación Secundaria de la Comunidad de Madrid, se sigue lo expuesto por Heinemann (2003) y Madella (2003) que explican que el deporte no es una industria o una rama industrial, por ello los puestos de trabajo referidos a la actividad física y el deporte no se recogen estadísticamente de forma específica, y como tal, estos datos han de deducirse de diversos cálculos, encuestas y estadísticas.

Por ello, tal y como expone Martínez del Castillo (1991), es necesario la utilización de listados indirectos para establecer el universo de trabajo para dicha población de estudio, ya que la identificación y localización de las unidades de las que se obtiene la información es muy compleja y difícil. Los listados utilizados en esta investigación han sido:

- Listado de Centros educativos de Educación Secundaria de la Comunidad de Madrid dentro de la Guía de centros docentes de la Comunidad de Madrid (Comunidad de Madrid, 2008a).

- Listado de Municipios y población de la Comunidad de Madrid en el año 2007 (Instituto de Estadística de la Comunidad de Madrid, 2007).

- Relación de personas que trabajan en el Programa Campeonatos Escolares de la Comunidad de Madrid (Comunidad de Madrid, 2008b).

A partir de estos listados y de los valores obtenidos se calcula un total de 1.650 personas que desarrollan la función de docencia en las actividades físico-deportivas extraescolares en centros educativos de Educación Secundaria de la Comunidad de Madrid.

La muestra registra las siguientes características: el tamaño de la muestra final fue de 350 docentes de las actividades físico-deportivas extraescolares en los centros educativos de Educación Secundaria en la Comunidad de Madrid (28\% mujeres y 72\% hombres; 61,2\% menores de 30 años y 38,8\% mayores de 30 años). Para el cálculo del tamaño de la muestra se consideraron varios aspectos: la población era finita; se recurre en la varianza poblacional al supuesto más desfavorable donde "P" y "Q" eran iguales con el $50 \%$ cada uno; el intervalo de confianza es del 95,5\%, con un margen de error de $\pm 4,75 \%$.

La afijación de la muestra ha sido proporcional a la distribución de las personas, según tamaño demográfico de los municipios y según la situación geográfica de los diferentes municipios de la Comunidad de Madrid respecto a las cinco áreas territoriales de esta Comunidad Autónoma. Además, se realizaron diversos submuestreos para dispersar la muestra y que influyese de forma positiva en la precisión de las estimaciones (Rodríguez, 2002). También se estableció una afijación proporcional en función de la titularidad de los centros educativos (público y privado), debido a que podrían encontrarse diferencias importantes en los resultados en función de su titularidad.

Se decidió entrevistar como máximo a dos personas objeto de estudio en cada centro educativo, siguiendo para ello diferentes investigaciones como las de Almodóvar y Maqueda (2001), Pablos (2006) y Martínez (2007), donde se entrevistan a dos personas por cada entidad. Al realizar este procedimiento se dispersó la muestra e influyó positivamente en la precisión de las estimaciones, disminuyendo el error muestral (Rodríguez, 1991 y Cea, 2001). 
Gallardo-Pérez, J.M.; Espada-Mateos, M.; Santacruz-Lozano, J.A.; Clemente-Remón, A. (2013). Las características laborales del profesorado de las actividades físico-deportivas extraescolares en función de la edad y el sexo, dentro de la Comunidad de Madrid. RICYDE. Rev. int. cienc. deporte. 30(9), 23-37. http://dx.doi.org/10.5232/ricyde2013.03102

El tipo de muestreo utilizado ha sido el muestreo aleatorio por conglomerados, dentro del cual se ha utilizado un muestreo polietápico, estratificado en primera fase por conglomerados, ya que se ha seguido una secuencia de etapas (aplicándose un muestreo aleatorio en cada una de las etapas) de selección de unidades muestrales (conglomerados) de mayor a menor rango, hasta llegar a los individuos que constituyen la muestra (Latorre, Del Rincón y Arnal, 1997; Cea, 2001 y Rodríguez, 2002).

\section{Instrumento}

El instrumento de recogida de la información utilizado ha sido el elaborado por González (2008b). No obstante, este cuestionario ha sido adaptado para las actividades y niveles pertenecientes a la etapa de Educación Secundaria y por ello, se ha validado el mismo en dos fases: el juicio de expertos y el estudio piloto.

Inicialmente, la entrevista estandarizada por medio de cuestionario diseñada ha sido revisada por cinco personas expertas, doctores y licenciados en Educación Física o en Ciencias de la Actividad Física y del Deporte. Después se efectuó una prueba piloto en la que se realizó la entrevista a setenta personas, con características similares a la población que se iba a estudiar. Esta cantidad establece la validación de la propia entrevista, ya que la cantidad de personas a las que se les realizó la entrevista es suficiente para validar dicho cuestionario según lo expuesto, entre otros autores, por Visauta (1989), Azorín y Sánchez-Crespo (1994), Cea (2001) o García Ferrando (2002).

Por tanto, la herramienta utilizada para recoger la información ha sido la entrevista estructurada por medio de cuestionario, la cual se compone de 55 preguntas cerradas que recogen cinco dimensiones relacionadas con: las características estructurales y organizativas de las actividades físico-deportivas extraescolares, las características sociodemográficas de las personas que trabajan en estas actividades(preguntas del cuestionario: 1-4), así como las características laborales, los aspectos didácticos y las características formativas de estas personas.

Para la realización del presente estudio, de todo el cuestionario se seleccionaron las dimensiones de las características sociodemográficas de las personas que trabajan en estas actividades y las características laborales(preguntas del cuestionario 43, 44, 47 y 48), cuyas variables son tipo de relación contractual e ingresos, así como los ítems relacionados con los objetivos del estudio.

\section{Procedimiento}

Esta investigación ha sido de corte transversal (Thomas y Nelson, 2007) debido a que la obtención de la información se desarrolla en un único periodo en el tiempo: el curso académico 2008-2009, y más concretamente entre los meses de octubre de 2008 a mayo de 2009, ya que las actividades físico-deportivas extraescolares en los centros educativos se suelen realizar en este periodo de tiempo.

Las 350 entrevistas de la muestra fueron realizadas por una única entrevistadora de forma personal (cara a cara), este procedimiento favorece que las respuestas sean de mayor calidad y espontaneidad (Lyberg, Biemer, De Leeuw, Dippo, y Trewin, 1997 y Cea, 2001). 
Gallardo-Pérez, J.M.; Espada-Mateos, M.; Santacruz-Lozano, J.A.; Clemente-Remón, A. (2013). Las características laborales del profesorado de las actividades físico-deportivas extraescolares en función de la edad y el sexo, dentro de la Comunidad de Madrid. RICYDE. Rev. int. cienc. deporte. 30(9), 23-37. http://dx.doi.org/10.5232/ricyde2013.03102

\section{Análisis de datos}

El análisis de datos ha sido efectuado tras ser tabulados y mecanizados éstos informáticamente. Se ha realizado un análisis de tablas de contingencia que incluyen las frecuencias absolutas, el valor de Chi-cuadrado de Pearson y su significación, así como el coeficiente de correlación Phi. Para ello se ha empleando el paquete de programas SPSS para Windows (V 15.0).

\section{Resultados}

\section{Situación contractual en función de las variables demográficas}

A continuación, en la tabla 1 y 2 se expondrán los tipos de contratos que tienen las personas que trabajan como docentes de actividades físico-deportivas extraescolares en función del sexo y de la edad. Cabe señalar que de las 350 personas que fueron entrevistadas únicamente 46 afirmaron tener algún tipo de contrato.

Así, en referencia a los datos obtenidos en función del tipo de contrato y el sexo de la población estudiada, en la Tabla 1 se observa que más de tres cuartas partes del total de hombres y mujeres tiene un contrato de tipo temporal. No obstante cabe señalar que existe una relación baja, pero no es estadísticamente significativa $[\mathrm{x} 2(1)=.016 ; \mathrm{p}=.900 ; \Phi=.019]$.

Tabla 1. Distribución de la contratación indefinida y temporal de las personas contratadas según sexo.

\begin{tabular}{|l|c|c|c|c|c|c|}
\hline \multirow{4}{*}{ Sexo } & \multicolumn{5}{|c|}{ Tipo de Contrato } & \multicolumn{2}{c|}{ Total } \\
& \multicolumn{2}{|c|}{ Temporal } & \multicolumn{2}{c|}{ Indefinido } & \multicolumn{2}{c|}{ Total } \\
\cline { 2 - 7 } Hombre & 23 & 76.6 & 7 & 23.3 & 30 & 100.0 \\
\cline { 2 - 7 } Mujer & 12 & 75 & 4 & 25 & 16 & 100.0 \\
\cline { 2 - 7 } Total & 35 & 76 & 11 & 24 & 46 & 100.0 \\
\hline
\end{tabular}

En la tabla 2, se aprecia que las personas que trabajan en el sector de actividades físicodeportivas extraescolares en secundaria tienen entre 20-24 años y tienen un contrato principalmente temporal. Además, existe una relación moderada y estadísticamente significativa entre la edad y la tipología del contrato $[\mathrm{x} 2(6)=17.462 ; \mathrm{p}=.008 ; \Phi=.616]$.

Tabla 2. Distribución de la contratación indefinida y temporal de las personas contratadas según edad.

\begin{tabular}{|c|c|c|c|c|c|c|c|c|c|c|c|c|c|c|}
\hline \multirow{3}{*}{$\begin{array}{l}\text { Tipo de } \\
\text { Contrato }\end{array}$} & \multicolumn{14}{|c|}{ Edad } \\
\hline & \multicolumn{2}{|c|}{ 16-19 } & \multicolumn{2}{|c|}{$20-24$} & \multicolumn{2}{|c|}{$25-29$} & \multicolumn{2}{|c|}{$30-\overline{34}$} & \multicolumn{2}{|c|}{$40-44$} & \multicolumn{2}{|c|}{$50-54$} & \multicolumn{2}{|c|}{ Total } \\
\hline & $\mathrm{n}$ & $\%$ & $\mathrm{n}$ & $\%$ & $\mathrm{n}$ & $\%$ & $\mathrm{n}$ & $\%$ & $\mathrm{n}$ & $\%$ & $\mathrm{n}$ & $\%$ & $\mathrm{n}$ & $\%$ \\
\hline Temporal & 1 & 2.7 & 15 & 42.8 & 8 & 22.8 & 5 & 14.2 & 3 & 14.5 & 1 & 2.7 & 35 & 76.1 \\
\hline Indefinido & 1 & 9 & 0 & 0 & 1 & 9 & 3 & 27.3 & 0 & 0 & 1 & 9 & 11 & 23.9 \\
\hline Total & 2 & 11.8 & 15 & 42.8 & 9 & 31.8 & 8 & 41.5 & 3 & 14.5 & 2 & 11.7 & 46 & 100.0 \\
\hline
\end{tabular}

\section{Experiencia profesional en función de las variables demográficas}

En la tabla 3 se puede observar que el mayor porcentaje, tanto en hombres como en mujeres, es el tiempo trabajado entre 1 y 3 años, seguido por menos de 1 año y entre 3 y 5 años en 
Gallardo-Pérez, J.M.; Espada-Mateos, M.; Santacruz-Lozano, J.A.; Clemente-Remón, A. (2013). Las características laborales del profesorado de las actividades físico-deportivas extraescolares en función de la edad y el sexo, dentro de la Comunidad de Madrid. RICYDE. Rev. int. cienc. deporte. 30(9), 23-37. http://dx.doi.org/10.5232/ricyde2013.03102

ambos sexos. Además, la relación entre el tiempo trabajado y el sexo es baja y no es significativa [x2 (4) $=4.141 ; \mathrm{p}=.387 ; \Phi=.109]$.

Tabla 3. Años que el profesorado lleva trabajando en las entidades para las cuales trabaja actualmente según el sexo.

\begin{tabular}{|c|c|c|c|c|c|c|}
\hline & \multicolumn{6}{|c|}{ Sexo } \\
\hline Tiempo trabajado & \multicolumn{2}{|c|}{ Hombre } & \multicolumn{2}{|c|}{ Mujer } & \multicolumn{2}{c|}{ Total } \\
\hline & $\mathrm{n}$ & $\%$ & $\mathrm{n}$ & $\%$ & $\mathrm{n}$ & $\%$ \\
\hline Menos de 1 año & 55 & 21.8 & 30 & 30.6 & 85 & 24.3 \\
\hline Entre 1 y 3 años & 100 & 39.7 & 35 & 35.7 & 135 & 38.6 \\
\hline Entre 3 y 5 años & 57 & 22.6 & 23 & 23.5 & 80 & 22.8 \\
\hline Entre 5 y 10 años & 32 & 12.7 & 8 & 8.2 & 40 & 11.4 \\
\hline Más de 10 años & 8 & 3.2 & 2 & 2 & 10 & 2.9 \\
\hline Total & 252 & 100.0 & 98 & 100.0 & 350 & 100.0 \\
\hline
\end{tabular}

Tal y como se observa en la tabla 4, la franja de edad donde más personas trabajan en el sector de la actividad física extraescolar es desde los 20 años hasta los 40. Este rango de edad coincide con el segmento de población donde más personas trabajan en función de su experiencia profesional anterior, es decir, partiendo de meses hasta los 5 años. Existe una relación moderada entre ambas variables y estadísticamente significativa [x2 (36) =79.047; $\mathrm{p}=.000 ; \Phi=.475]$.

Tabla 4. Años que el profesorado lleva trabajando en las entidades para las cuales trabaja actualmente según la edad.

\begin{tabular}{|c|c|c|c|c|c|c|c|c|c|c|c|c|c|c|c|c|c|c|c|c|c|c|}
\hline \multirow{3}{*}{$\begin{array}{l}\text { Tiempo } \\
\text { trabajado }\end{array}$} & \multicolumn{22}{|c|}{$\underline{\text { Edad }}$} \\
\hline & \multicolumn{2}{|c|}{$16-19$} & \multicolumn{2}{|c|}{$20-24$} & \multicolumn{2}{|c|}{$25-29$} & \multicolumn{2}{|c|}{$30-34$} & \multicolumn{2}{|c|}{$35-39$} & \multicolumn{2}{|c|}{$40-44$} & \multicolumn{2}{|c|}{$45-49$} & \multicolumn{2}{|c|}{$50-54$} & \multicolumn{2}{|c|}{$55-59$} & \multicolumn{2}{|c|}{ Más 60} & \multicolumn{2}{|c|}{ Total } \\
\hline & $\mathrm{n}$ & $\%$ & $\mathrm{n}$ & $\%$ & $\mathrm{n}$ & $\%$ & $\mathrm{n}$ & $\%$ & $\mathrm{n}$ & $\%$ & $\mathrm{n}$ & $\%$ & $\mathrm{n}$ & $\%$ & $\mathrm{n}$ & $\%$ & $\mathrm{n}$ & $\%$ & $\mathrm{n}$ & $\%$ & $\mathrm{n}$ & $\%$ \\
\hline $\begin{array}{l}\text { Menos } \\
\text { de } 1 \text { año }\end{array}$ & 13 & 41.9 & 34 & 28.8 & 16 & 24.6 & 11 & 18.6 & 5 & 17.8 & 1 & 5.9 & 2 & 12.5 & 1 & 10 & 2 & 40 & 0 & 0 & 85 & 24.3 \\
\hline $\begin{array}{l}\text { Entre } 1 \mathrm{y} \\
3 \text { años }\end{array}$ & 15 & 48.5 & 53 & 44.9 & 21 & 32.3 & 26 & 44.1 & 7 & 25 & 6 & 35.3 & 5 & 31.2 & 2 & 20 & 0 & 0 & 0 & 0 & 135 & 38.6 \\
\hline $\begin{array}{c}\text { Entre } 3 \text { y } \\
5 \text { años }\end{array}$ & 3 & 9.6 & 21 & 17.7 & 17 & 26.1 & 12 & 20.3 & 9 & 32.1 & 7 & 41.1 & 5 & 31.2 & 3 & 30 & 2 & 40 & 1 & 100 & 80 & 22.8 \\
\hline $\begin{array}{c}\text { Entre } 5 \mathrm{y} \\
10 \text { años }\end{array}$ & 0 & 0 & 10 & 8.6 & 10 & 15.4 & 8 & 13.5 & 5 & 17.8 & 2 & 11.8 & 4 & 25 & 1 & 10 & 0 & 0 & 0 & 0 & 40 & 11.4 \\
\hline $\begin{array}{l}\text { Más de } \\
10 \text { años }\end{array}$ & 0 & 0 & 0 & 0 & 1 & 1.6 & 2 & 3.5 & 2 & 7.3 & 1 & 5.9 & 0 & 0 & 3 & 30 & 1 & 20 & 0 & 0 & 10 & 2.9 \\
\hline Total & 31 & 100.0 & 118 & 100.0 & 65 & 100.0 & 59 & 100.0 & 28 & 100.0 & 17 & 100.0 & 16 & 100.0 & 10 & 100.0 & 5 & 100.0 & 1 & 100.0 & 350 & 100.0 \\
\hline
\end{tabular}

Salario en función del sexo y el tipo de centro educativo.

Los datos extraídos de la tabla 5 muestran que el porcentaje donde menor salario se recibe es por parte de los centros educativos privados, en cambio el porcentaje donde más salario se recibe es en los centros educativos públicos. Existe una relación alta y significativa entre el 
Gallardo-Pérez, J.M.; Espada-Mateos, M.; Santacruz-Lozano, J.A.; Clemente-Remón, A. (2013). Las características laborales del profesorado de las actividades físico-deportivas extraescolares en función de la edad y el sexo, dentro de la Comunidad de Madrid. RICYDE. Rev. int. cienc. deporte. 30(9), $23-37$. http://dx.doi.org/10.5232/ricyde2013.03102

salario recibido y el tipo de centro educativo donde se trabaje $[\mathrm{x} 2(14)=185.922 ; \mathrm{p}=.000$; $\Phi=.745]$.

Tabla 5. Porcentaje de ingresos totales mensuales de las personas en función del tipo de centro educativo.

\begin{tabular}{|c|c|c|c|c|c|c|c|c|c|c|c|c|c|c|c|c|c|c|}
\hline \multirow{3}{*}{$\begin{array}{l}\text { Tipo de } \\
\text { Colegio }\end{array}$} & \multicolumn{18}{|c|}{ Ingresos Mensuales } \\
\hline & \multicolumn{2}{|c|}{$\begin{array}{c}\text { Menos } \\
150 €\end{array}$} & \multicolumn{2}{|c|}{$\begin{array}{c}\text { Entre } 150 € \\
\text { y } 300 €\end{array}$} & \multicolumn{2}{|c|}{$\begin{array}{c}\text { Entre } 300 € \\
\text { у } 490 €\end{array}$} & \multicolumn{2}{|c|}{$\begin{array}{c}\text { Entre } \\
490 € \mathrm{y} \\
630 €\end{array}$} & \multicolumn{2}{|c|}{$\begin{array}{c}\text { Entre } \\
799 € \mathrm{y} \\
1052 €\end{array}$} & \multicolumn{2}{|c|}{$\begin{array}{c}\text { Entre } \\
1500 € \mathrm{y} \\
1953 €\end{array}$} & \multicolumn{2}{|c|}{$\mathrm{Ns} / \mathrm{Nc}$} & \multicolumn{2}{|c|}{ Gratis } & \multicolumn{2}{|c|}{ Total } \\
\hline & $\mathrm{n}$ & $\%$ & $\mathrm{n}$ & $\%$ & $\mathrm{n}$ & $\%$ & $\mathrm{n}$ & $\%$ & $\mathrm{n}$ & $\%$ & $\mathrm{n}$ & $\%$ & $\mathrm{n}$ & $\%$ & $\mathrm{n}$ & $\%$ & $\mathrm{n}$ & $\%$ \\
\hline Público & 1 & 0.5 & 100 & 45.6 & 117 & 53.4 & 0 & 0 & 1 & 0.5 & 0 & 0 & 0 & 0 & 0 & 0 & 219 & 100.0 \\
\hline Privado & 5 & 12.8 & 21 & 53.8 & 3 & 7.7 & 0 & 0 & 2 & 5.2 & 1 & 2.5 & 6 & 15.5 & 1 & 2.5 & 39 & 100.0 \\
\hline Concertado & 25 & 32.4 & 40 & 52 & 8 & 10.4 & 4 & 5.2 & 0 & 0 & 0 & 0 & 0 & 0 & 0 & 0 & 77 & 100.0 \\
\hline Total & 31 & 9.3 & 161 & 48.1 & 128 & 38.2 & 4 & 1.1 & 3 & 0.8 & 1 & 0.3 & 6 & 1.9 & 1 & 0.3 & 335 & 100.0 \\
\hline
\end{tabular}

Respecto a los ingresos mensuales de las personas que trabajan en las actividades físicodeportivas extraescolares, en la Tabla 6 se muestra que a grandes rasgos los ingresos son similares en ambos sexos. No obstante, si se profundiza en los resultados presentados, se observa que las mujeres presentan mayor porcentaje que los hombres al percibir ingresos menores de $150 €$ mensuales, y que los hombres presentan un porcentaje más elevado al percibir entre 300 y $490 €$. Además, existe una relación moderada y estadísticamente significativa entre ambas variables $[\mathrm{x} 2(7)=15.943 ; \mathrm{p}=.026 ; \Phi=.218]$.

Tabla 6. Porcentaje de ingresos totales mensuales de las personas en función del sexo.

\begin{tabular}{|c|c|c|c|c|c|c|c|c|c|c|c|c|c|c|c|c|c|c|}
\hline \multirow[t]{3}{*}{ Sexo } & \multicolumn{18}{|c|}{ Ingresos Mensuales } \\
\hline & \multicolumn{2}{|c|}{$\begin{array}{l}\text { Menos } \\
150 €\end{array}$} & \multicolumn{2}{|c|}{$\begin{array}{c}\text { Entre } 150 € \\
\text { у } 300 €\end{array}$} & \multicolumn{2}{|c|}{$\begin{array}{c}\text { Entre } 300 € \\
\text { y } 490 €\end{array}$} & \multicolumn{2}{|c|}{$\begin{array}{c}\text { Entre } \\
490 € \mathrm{y} \\
630 €\end{array}$} & \multicolumn{2}{|c|}{$\begin{array}{c}\text { Entre } \\
799 € \mathrm{y} \\
1052 €\end{array}$} & \multicolumn{2}{|c|}{$\begin{array}{c}\text { Entre } \\
1500 € \mathrm{y} \\
1953 €\end{array}$} & \multicolumn{2}{|c|}{$\mathrm{Ns} / \mathrm{Nc}$} & \multicolumn{2}{|c|}{ Gratis } & \multicolumn{2}{|c|}{ Total } \\
\hline & $\mathrm{n}$ & $\%$ & $\mathrm{n}$ & $\%$ & $\mathrm{n}$ & $\%$ & $\mathrm{n}$ & $\%$ & $\mathrm{n}$ & $\%$ & $\mathrm{n}$ & $\%$ & $\mathrm{n}$ & $\%$ & $\mathrm{n}$ & $\%$ & $\mathrm{n}$ & $\%$ \\
\hline Hombre & 16 & 6.5 & 117 & 47.7 & 101 & 41.3 & 2 & 0.8 & 2 & 0.8 & 0 & 0 & 6 & 2.5 & 1 & 0.4 & 245 & 100.0 \\
\hline Mujer & 15 & 16.7 & 44 & 48.9 & 27 & 30 & 2 & 2.2 & 1 & 1.1 & 1 & 1.1 & 0 & 0 & 0 & 0 & 90 & 100.0 \\
\hline Total & 31 & 9.2 & 161 & 48 & 128 & 38.2 & 4 & 1.2 & 3 & 0.9 & 1 & 0.3 & 0 & 1.8 & 1 & 0.4 & 335 & 100.0 \\
\hline
\end{tabular}

\section{Discusión}

En la presente investigación se ha obtenido que de las 350 personas entrevistadas, únicamente $46(13.1 \%)$ afirmaron tener algún tipo de contrato. Estos resultados son más alarmantes que los obtenidos en otras investigaciones. Así, Campos (2005) obtiene que más del 50\% de estas personas no tiene ningún tipo de contratación y en la investigación llevada a cabo por Álamo (2004), estas personas representan el 68.4\%. Por su parte, en el estudio de Martínez del Castillo (1991) las personas contratadas representaban el 16\% del total.

En el presente trabajo se ha obtenido que las personas que trabajan en él ámbito de las actividades físico-deportivas extraescolares tienen un contrato eminentemente de carácter temporal, presentando los hombres un porcentaje del $76.6 \%$ y las mujeres del $75 \%$. A pesar 
Gallardo-Pérez, J.M.; Espada-Mateos, M.; Santacruz-Lozano, J.A.; Clemente-Remón, A. (2013). Las características laborales del profesorado de las actividades físico-deportivas extraescolares en función de la edad y el sexo, dentro de la Comunidad de Madrid. RICYDE. Rev. int. cienc. deporte. 30(9), 23-37. http://dx.doi.org/10.5232/ricyde2013.03102

de que estos datos son muy preocupantes, son ligeramente más esperanzadores que los obtenidos en investigaciones anteriores. Así, Campos (2005) obtuvo que más del $90 \%$ de las personas que trabajan en actividades físico-deportivas extraescolares tienen contratos temporales y menos del 10\% poseen una contratación indefinida. Asimismo, González (2008a) expone que la gran mayoría de las personas que trabajan como docentes de actividades físico-deportivas extraescolares y tienen contrato, poseen contratos de carácter temporal $(83,4 \%)$, siendo sólo un $16,6 \%$ las personas que tienen un contrato de tipo indefinido.

Por su parte, Martínez y col. (2008) obtienen porcentajes muy similares a los de la presente investigación, ya que el $75 \%$ de los docentes de actividades físico-deportivas extraescolares que tienen algún tipo de contrato es de carácter temporal, y tan sólo un $25 \%$ presenta contratación indefinida.

La edad de estas personas tiende a ser baja, siendo el porcentaje de mayor representación la edad comprendida entre los 20-24 años, al igual que en el estudio de Gallardo y Campos (2011) donde 48,3\% de las personas que trabajan está comprendido dentro de ese rango de edad. Así, en la presente investigación se ha obtenido que un 57,8\% de las personas de este rango de edad tienen un contrato de carácter temporal. Estos datos son inferiores a los presentados por González (2008a), en cuyo estudio el 95\% de las personas de entre 20 y 24 años tienen contratos de tipo temporal.

Ante esta situación, diversos autores, como Campos y Viaño (1999), Montalvo (2008), Palomar (2000) y Rebollo y Sánchez (2000) manifiestan que mediante la articulación de un adecuado desarrollo legislativo y laboral donde se expongan unas adecuadas condiciones laborales por parte de los diferentes agentes empleadores, ayudaría a solucionar muchos de los problemas que existen actualmente en el ámbito de la actividad física y el deporte.

Además, se ha observado que la experiencia profesional no varía en función del sexo, ya que existen porcentajes muy similares entre hombres y mujeres. Además, el tiempo trabajado por parte de las personas que desarrollan la función de docencia de actividades físico-deportivas extraescolares no supera, en la mayoría de los casos, los 5 años. En este sentido, se observa que la movilidad laboral dentro de este campo es alta, ya que el porcentaje de mayor proporción es la franja de entre 1 y 3 años (38,6\%, hombres y mujeres), al igual que en los estudios Campos (2005), donde el 70\% de las personas lleva trabajando menos de 3 años y Saura (1996), donde el 23,5\% de los entrenadores lleva trabajando menos de un año.

Por otra parte, no se encuentra evidencia porcentual de que exista una diferencia de sueldo en función del sexo dentro del presente estudio. Se debe destacar que los salarios son bajos, es decir, el mayor porcentaje se observa entre $150 €$ y $300 €$, con un $48,1 \%$ y entre $300 €$ y $490 €$ con un $38,2 \%$. Asimismo, resulta interesante señalar que los centros educativos privadosconcertados son los que presentan el mayor porcentaje de ingresos menores a $150 €$. Sin embargo, son estos centros los que presentan mayores porcentajes en cuanto a la retribución de salarios más altos, aunque dichos porcentajes son muy bajos. Estos resultados coinciden con los obtenidos en los estudios de Fraile (1996) y González (2008a), en los cuales, la mayoría de los entrenadores tiene unos ingresos mensuales inferiores a $300 €$.

En esta línea, Nuviala (2002) expone que muchas veces en el deporte escolar sólo se recibe una gratificación económica. Igualmente, Álamo (2004) manifiesta que el 47,4\% de las personas no recibe retribución económica por realizar esta función. Finalmente, Nuviala 
Gallardo-Pérez, J.M.; Espada-Mateos, M.; Santacruz-Lozano, J.A.; Clemente-Remón, A. (2013). Las características laborales del profesorado de las actividades físico-deportivas extraescolares en función de la edad y el sexo, dentro de la Comunidad de Madrid. RICYDE. Rev. int. cienc. deporte. 30(9), 23-37. http://dx.doi.org/10.5232/ricyde2013.03102

(2002) expone que la compensación económica por hora de trabajo es desigual, ya que varía entre 3 y 9 euros la hora.

Teniendo en cuenta los resultados obtenidos en el presente estudio, se puede afirmar que las personas que trabajan en actividades físico-deportivas extraescolares en la Comunidad de Madrid presentan una precaria situación contractual, y que además son las personas más jóvenes las que sufren esta situación en mayor medida. Asimismo, es una función laboral que tiende a realizarse, en general, durante un breve periodo de tiempo y que su remuneración mensual es baja. Todo ello indica que estamos atendiendo a una función laboral que no está suficientemente reconocida y valorada. Así, profesores, padres y madres, instituciones, etc. debemos plantearnos que si las personas encargadas de la formación físico-deportiva de nuestros escolares fuera del horario lectivo no se sienten reconocidas, probablemente su implicación será menor, y este aspecto repercutirá inevitablemente en la calidad del proceso de enseñanza/aprendizaje de este tipo de actividades.

Esta precaria situación, contrasta con las reflexiones expuestas por diferentes autores, como Bocarro, Kansters, Casper \& Forrester (2008), Carranza (2008), Cohen, Taylor, Zonta, Vestal \& Schuster (2007), Hernández y Velázquez (1996), Montesinos (2005), Orts y Mestre (2005), Pabayo, O'Loughlin, Gauvin, Paradis \& Gray-Donald (2006) y Trudeau y Shephard (2005), que manifiestan la gran importancia que tienen el deporte escolar y las actividades físicodeportivas extraescolares para la educación y la salud integral del alumnado en edad escolar. Si se comparan los resultados obtenidos en la presente investigación con la opinión de los autores citados anteriormente, se puede corroborar que existe una gran incoherencia en el ámbito de las actividades físico-deportivas extraescolares, causada por la gran inestabilidad y precariedad del profesorado, así como por la poca importancia que se le otorga en la práctica a este tipo de actividades.

\section{Conclusiones}

La situación laboral de las personas que trabajan en funciones de actividad físico-deportiva extraescolar es bastante precaria, ya que la mayoría de los contratos son de duración temporal, tanto en el caso de los hombres como en el de las mujeres. Esta situación afecta principalmente a los jóvenes, debido a que la mayoría de las personas que trabaja en este sector tiene entre 20 y 29 años, es decir, un grupo de población que se encuentra con contratos temporales y cuya experiencia profesional no supera los 5 años. A todo ello se suma que los ingresos mensuales más altos a los que puede optar un trabajador dentro de este ámbito son de 500 euros.

Todo ello demuestra que estamos ante una situación de desprotección laboral y social del trabajador y bajo una gran economía sumergida. 
Gallardo-Pérez, J.M.; Espada-Mateos, M.; Santacruz-Lozano, J.A.; Clemente-Remón, A. (2013). Las características laborales del profesorado de las actividades físico-deportivas extraescolares en función de la edad y el sexo, dentro de la Comunidad de Madrid. RICYDE. Rev. int. cienc. deporte. 30(9), 23-37. http://dx.doi.org/10.5232/ricyde2013.03102

\section{Referencias Bibliográficas}

Álamo, J. M. (2001). Análisis del deporte escolar en la isla de Gran Canaria. Hacia un modelo de deporte escolar. Tesis Doctoral. Facultad de Ciencias de la Actividad Física y el Deporte, Universidad de Las Palmas de Gran Canaria. Las Palmas.

Álamo, J. M. (2004). El deporte escolar en Canarias. En A. Fraile (Coord). El deporte escolar en el siglo XXI: Análisis y debate desde una perspectiva europea. (pp. 133152). Barcelona: Editorial Graó.

Álamo, J. M.; Amador, F., y Pintor, P. (2002). El deporte escolar: conquista de nuevos espacios en el mercado laboral. Revista Española de Educación Física y Deportes, 4, 510.

Almodóvar, A., y Maqueda, J. (2001). IV Encuesta nacional de condiciones de trabajo. Madrid: Instituto Nacional de Seguridad e Higiene en el Trabajo.

Alvira, F. (2002). Diseños de investigación social: Criterios operativos. En F. Alvira, M. García Ferrando y J. Ibáñez (Comps.). El análisis de la realidad social. Métodos y técnicas de investigación ( $3^{a}$ ed.) (pp. 99-125). Madrid: Alianza editorial.

Azorín, F., y Sánchez-Crespo J.L. (1994). Métodos y aplicaciones de muestreo. Madrid: Alianza editorial.

Bocarro, J.; Kanters, M. A.; Casper, J. \& Forrester, S. (2008). School Physical Education, Extracurricular Sports and Lifelong Active Living. Journal of Teaching in Physical Education, 27, 155-166.

Campos, A. (2001). La situación actual de los profesionales de la actividad física y el deporte en las instalaciones deportivas de la Comarca Burgalesa de la Ribera del Duero. Trabajo de investigación. Facultad de Ciencias de la Actividad Física y del deporte de Valencia, Departamento de Educación Física y Deportiva, Universidad de Valencia. Valencia.

Campos, A. (2005). Situación profesional de las personas que trabajan en funciones de actividad física y deporte en la Comunidad Autónoma Valenciana (2004). Tesis doctoral. Facultad de Ciencias de la Actividad Física y del Deporte, Departamento de Educación Física y Deportiva, Universidad de Valencia. Valencia.

Camy, J. (2006). Employment opportunities in the sports sector: a review of the European situation. En Beyond the Scoreboard. Youth Employment Opportunities \& Skills Development in the Sport Sector. (pp. 85-104). Genova: Giovani di Cola.

Carranza, M. (2008). Deporte escolar y educación: El nuevo modelo de deporte en edad escolar de la ciudad de Barcelona. En A. I. Hernández, L. F. Martínez y C. Águila (Eds.). El deporte escolar en la sociedad contemporánea. (pp. 89-101). Almería: Universidad de Almería.

Carratalá, V. (2011). Cualificación y perfil profesional en el deporte escolar. II Congreso del deporte en Edad Escolar. UIRFIDE, Universidad de Valencia, Valencia.

Carratalá, V.; Mayorga, J.; Mestre, J.; Montesinos, J., y Rubio, S. (2011) Estudio del mercado laboral y de las competencias profesionales del titulado. Universidad del País Vasco. Disponible en: http://www.kirolang.org, (Consultado el 11/01/2012).

Cea D'ancona, M. A. (2001). Metodología cuantitativa. Estrategias y técnicas de investigación social. Madrid: Síntesis.

Cohen. D. A.; Taylor, S. L.; Zonta, M.; Vestal, K. D., \& Schuster, M. A. (2007). Availability of high school extracurricular sports programs and high-risk behaviors. J ournal of School Health, 77 (2), 80-86. 
Gallardo-Pérez, J.M.; Espada-Mateos, M.; Santacruz-Lozano, J.A.; Clemente-Remón, A. (2013). Las características laborales del profesorado de las actividades físico-deportivas extraescolares en función de la edad y el sexo, dentro de la Comunidad de Madrid. RICYDE. Rev. int. cienc. deporte. 30(9), 23-37. http://dx.doi.org/10.5232/ricyde2013.03102

Comisiones Obreras (2000). Informe cuantitativo y cualitativo de gimnasios y piscinas. Disponible en: http://www.revista.consumer.es, (Consultado el 12/11/2011).

Comunidad de Madrid (2007). Listado de Municipios y población de la Comunidad de Madrid. Disponible en: http://es.wilkipedia.org/wilki/Lista_de_municipios_de_Madrid, (Consultado el 16/12/2011).

Comunidad de Madrid (2008a). Programa Deportivo Escolar Madrid Olímpico. Disponible en: www.madrid.org, (Consultado el 12/11/2011).

Comunidad de Madrid (2008b). El Deporte Infantil de la Comunidad de Madrid. Disponible en: www.madrid.org, (Consultado el 12/11/2012).

Comunidad de Madrid (2008c). Campeonatos Escorares de la Comunidad de Madrid. Disponible en: www.campeonatosescolares.es, (Consultado el 13/12/2011).

Comunidad de Madrid (2008d). Guía de centros docentes de la Comunidad de Madrid. Disponible en: www.madrid.org/centros_docentes/guia/index.html, (Consultado el 13/12/2011).

De Diego, R.; Fraile, A., \& Boada, J. (2004). Training needs among sport technicians. Psicothema, 16 (1), 84-87.

Delgado-Noguera, M.A. (2000). Análisis de los comportamientos de los docentes en la enseñanza deportiva en edad escolar. En VV.AA (Eds.). I Congreso Nacional de deporte en edad escolar, (pp. 161-197). Dos Hermanas (Sevilla): Excmo Ayto de Dos Hermanas.

Fraile, A. (1996). Reflexiones sobre la presencia del deporte en la escuela. Revista de educación Física. Renovar la teoría y la práctica, 64, 5-10.

García Ferrando, M. (2002). La encuesta. En F. Alvira, M. García Ferrando y J. Ibáñez (Comps.), El análisis de la realidad social. Métodos y técnicas de investigación (ed. $3^{a}$ ) (pp. 141-170). Madrid: Alianza editorial

Garrigós, F. (2002). La regulación del ejercicio de la profesión como activo en el desarrollo de la economía del deporte. Actividad física. Ciencia y profesión, 2, 5-8.

Gómez, O. (2001). El mercado de trabajo en el sector deportivo en España y en Europa. Revista J urídica del deporte, 5, 69-96.

González, M. D. (2008a). El deporte escolar en la comunidad autónoma de Madrid: Intervención didáctica y recursos humanos en las actividades físico-deportivas extraescolares en los centros educativos. Tesis Doctoral. Facultad de Ciencias de la Actividad Física y del Deporte, Departamento de Educación Física y Deportiva, Universidad de Valencia. Valencia.

González, M. D. (2008b). Elaboración y validación de una entrevista estandarizada por medio de cuestionario para el estudio del deporte escolar en los centros educativos de la Comunidad Autónoma de Madrid: la intervención didáctica y los recursos humanos en las actividades físico-deportivas extraescolares. Trabajo de investigación. Facultad de Ciencias de la Actividad Física y del deporte de Valencia, Departamento de Educación Física y Deportiva, Universidad de Valencia. Valencia.

Heinemann, K. (2003). Introducción a la metodología de la investigación empírica. Barcelona: Paidotribo.

Hernández, J. L., y Velázquez, R. (1996). La actividad física y deportiva extraescolar en los centros educativos. Madrid: MEC. 
Gallardo-Pérez, J.M.; Espada-Mateos, M.; Santacruz-Lozano, J.A.; Clemente-Remón, A. (2013). Las características laborales del profesorado de las actividades físico-deportivas extraescolares en función de la edad y el sexo, dentro de la Comunidad de Madrid. RICYDE. Rev. int. cienc. deporte. 30(9), 23-37. http://dx.doi.org/10.5232/ricyde2013.03102

Latorre, A.; Del Rincón, D., y Arnal, J. (1997). Bases metodológicas de la investigación. Barcelona: Hurtado Ediciones.

Lussier, R.N., \& Kimball, D.C. (2008). Applied sport management skills. Ed. Human Kinetics, Champaign.

Lyberg, L.; Biemer, P.; De Leeuw, E.; Dippo, C., \& Trewin, D. (1997). Survey Measurement and Process Quality. Canada: Wiley Series in Probability and Statistics.

Madella, A. (2003). Methods for analysing sports employment in Europe. Managing Leisure, 8 (2), 56-59.

Martínez del Castillo, J. (Dir.) (1991). La estructura ocupacional del deporte en España. Encuesta realizada sobre los sectores de Entrenamiento, Docencia, Animación y Dirección. Madrid: CSD.

Martínez, G. (2007). Los recursos humanos de la actividad física y del deporte en la Comunidad Autónoma Valenciana. Tesis Doctoral. Facultad de Ciencias de la Actividad Física y del Deporte, Departamento de Educación Física y Deportiva, Universidad de Valencia. Valencia.

Martínez, G.; Campos, A.; Pablos, C., y Mestre, J. A. (2008). Los recursos humanos de la actividad física y del deporte. Funciones y características socio-demográficas, laborales y formativas. Valencia: Tirant Lo Blanch.

Montesinos, J. M. (2005). El deporte en la edad escolar: Aspectos organizativos. En I Congrés d'Esport en edat escolar (pp. 50-59). Valencia: Ajuntament de València y Fundació Esportiva Municipal.

Mosquera, M. J.; Rivas, A., y Saavedra, M. (1998). Percepción profesional del alumno de ciencias de la Actividad Física y Deporte del I.N.E.F. de Galicia. En M. González, R. Martín, J. L. Salvador, J. Fernández y M. Bobo (Coords.), Educación Física e deporte no seculo XXI, I (pp. 253-265). A Coruña: Universidade da Coruña.

Nuviala, A. (2002). Las escuelas deportivas en el entorno rural del servicio comarcal de deportes "Corredor del Ebro" y el municipio Fuentes de Ebro. Tesis doctoral. Facultad de Ciencias de la Educación, Universidad de Huelva. Huelva.

Orts, F., y Mestre, J. A. (2005). La organización del deporte en edad escolar en la ciudad de Valencia desde una perspectiva educativa. En Ajuntament de València y Fundació Esportiva Municipal (ed). I Congrés d'Esport en edat escolar (pp.11-29). Valencia: Ajuntament de València y Fundació Esportiva Municipal.

Pabayo, R.; O 'Loughlin, J.; Gauvin, L.; Paradis, G., \& Gray-Donald, K. (2006). Effect of a Ban on Extracurricular Sports Activities by Secundary School Teachers on Physical Activity Levels of Adolescents. Health Education \& Behavior, 33 (5), 690-702.

Pablos, C. (2006). Empleo y Deporte: situación laboral de las personas que trabajan en actividad física y deporte en la Comunidad Valenciana". Valencia: Consellería de Cultura y Educación, CC.AA.- UIRFIDE.

Rodríguez, J. (1991). Métodos de muestreo. Madrid: CIS.

Rodríguez, J. (2002). La muestra: teoría y aplicación. En F. Alvira, M. García Ferrando y J. Ibáñez (Comps.). El análisis de la realidad social. Métodos y técnicas de investigación (ed. 3a) (pp. 445-482). Madrid: Alianza editorial.

Sánchez, A., y Rebollo, S. (2000) Situación del mercado laboral actual en el ámbito de la actividad física y del deportiva. Revista Motricidad, (6), 141-154. 
Gallardo-Pérez, J.M.; Espada-Mateos, M.; Santacruz-Lozano, J.A.; Clemente-Remón, A. (2013). Las características laborales del profesorado de las actividades físico-deportivas extraescolares en función de la edad y el sexo, dentro de la Comunidad de Madrid. RICYDE. Rev. int. cienc. deporte. 30(9), 23-37. http://dx.doi.org/10.5232/ricyde2013.03102

Saura, J. (1996). El entrenador en el deporte escolar. Lleida: Fundació Pública Institut d'Estudis Llerdencs.

Thomas, J. R., \& Nelson, J. K. (2007). Research Methods in Physical Activity. United Estates: Human Kinetics.

Trudeau, F., \& Shephard, R. J. (2005). Contribution of School Programmes to Physical Activity Levels and Attitudes in Children and Adults. Sports Medicine, 35 (2), 89-105.

Visauta, B. (1989). Técnicas de investigación social. I: recogida de datos. Barcelona: PPU. 